\title{
COUPLING MULTI-BODY AND FLUID DYNAMICS ANALYSIS WITH PRECICE AND MBDYN
}

\author{
CLAUDIO G. CACCIA* AND PIERANGELO MASARATI ${ }^{\dagger}$ \\ *Department of Aerospace Science and Technology, Politecnico di Milano \\ via La Masa 34, 20156, Milan, Italy \\ e-mail: claudiogiovanni.caccia@mail.polimi.it, web page: http://www.aero.polimi.it/ \\ ${ }^{\dagger}$ Department of Aerospace Science and Technology, Politecnico di Milano \\ via La Masa 34, 20156, Milan, Italy \\ e-mail: pierangelo.masarati@polimi.it, web page: http://www.aero.polimi.it/
}

Key words: Multibody System Dynamics, Cosimulation, Fluid-Structure Interaction

\begin{abstract}
.
The software library preCICE allows to connect single physics solvers to perform multiphysics cosimulations in a partitioned way. We interfaced preCICE with the multibody dynamics software MBDyn and assessed its performance with the set of well-known benchmarks proposed by Turek and Hron. The set-up consists of a laminar incompressible flow around a slender elastic object, which is suitable to be simulated via MBDyn beam elements connected to a cloud of interface points.
\end{abstract}

\section{MOTIVATION}

In this work, the Multibody Dynamics solver MBDyn ${ }^{1}$ [10] has been interfaced with the multiphysics coupling library preCICE [3] to extend MBDyn capabilities to solve fluid structure interaction (FSI) problems.

MBDyn is a free general-purpose Multibody Dynamics solver developed at the Department of Aerospace Science and Technology of Politecnico di Milano, which models the constrained nonlinear dynamics of rigid and flexible bodies formulated as sets of Differential-Algebraic Equations (DAEs) [10].

When FSI simulations involve slender and flexible structures, it is particularly interesting to use a reduced dimensionality finite element model (i.e. a set of beams or shells) along with a form of mapping between the interface (wet surface) and the structural model, especially when the two are topologically incompatible, in order to exchange the mutual kinematics and dynamics [12].

\footnotetext{
${ }^{1}$ https :/www.mbdyn.org/, last accessed May 2021.
} 


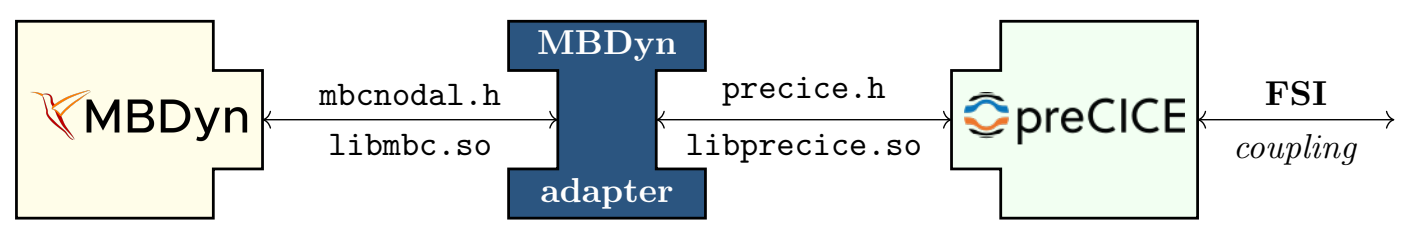

Figure 1: MBDyn adapter interface scheme

MBDyn models slender deformable components using an original, geometrically exact finite volume formulation for beam elements with a high level of flexibility $[8,1]$. MBDyn is also able to exchange kinematics and load information with a cloud of external points of arbitrary topology [12].

PreCICE is an increasingly popular open-source coupling library which provides the components to connect traditional single-physics solvers to generate a partitioned multiphysics simulation (e.g. fluid-structure interaction, conjugated heat transfer, solid-solid interaction, etc.).

The implementation of an adapter connecting MBDyn and preCICE represents an advantage and an extension of capabilities for both applications. On the one hand, various preCICE adapters for fluid solvers have already been developed. MBDyn can connect in a standardized way to a considerable number of codes, including many popular, wellvalidated open source and commercial one
simulation capabilities (e.g. [4]).
On the other hand, with a fully integrate
the opportunity to connect to a multibody
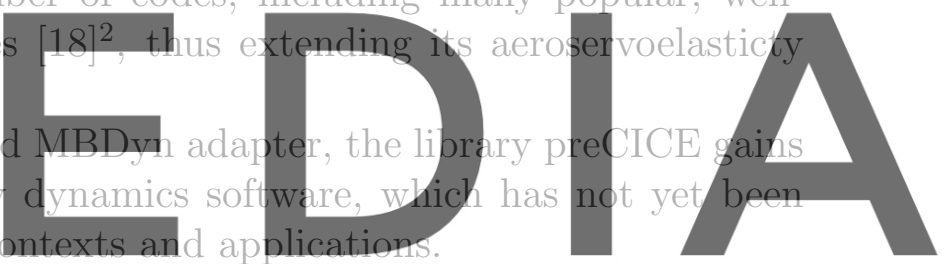

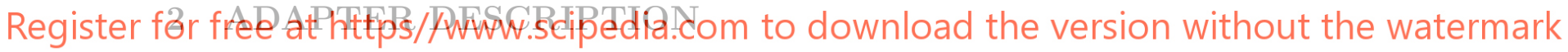

An adapter is the "glue-code" that allows the interface of an existing software component to be used by another component without modifying its source code. In the present case it is possible to exploit the APIs given by MBDyn and preCICE, so that the adapter itself is independent from both codes (see Fig. 1).

\subsection{MBDyn Features}

MBDyn can simulate linear and non-linear dynamics of rigid and flexible bodies (including geometrically exact and composite-ready beam and shell finite elements, component mode synthesis elements, lumped elements) subjected to kinematic constraints, external forces and control subsystems [10]. Nodes are the basic blocks of an MBDyn model: they instantiate kinematic degrees of freedom and the corresponding equilibrium equations. Elements constitute the components of the multi-body model. With minor exceptions, each of them connects one or more nodes. They write contributions to equations instanti-

\footnotetext{
${ }^{2}$ for an updated list see https://precice.org/adapters-overview.html
} 


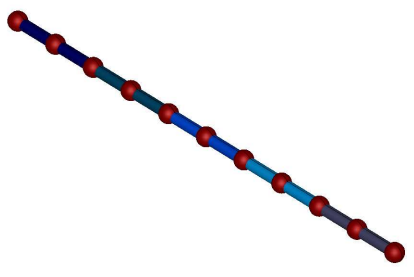

(a) Model: nodes and beams

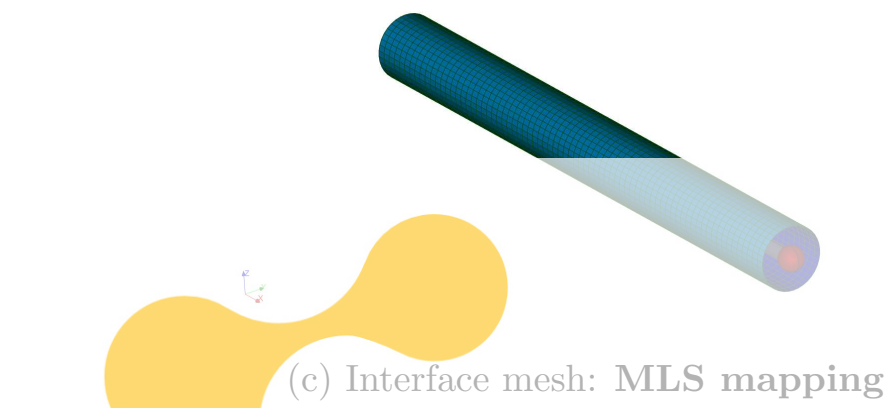

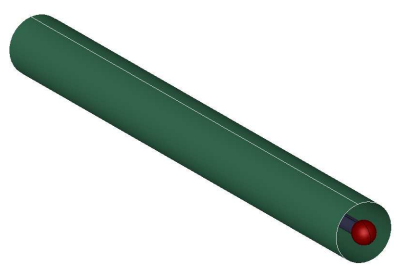

(b) Interface geometry: shape

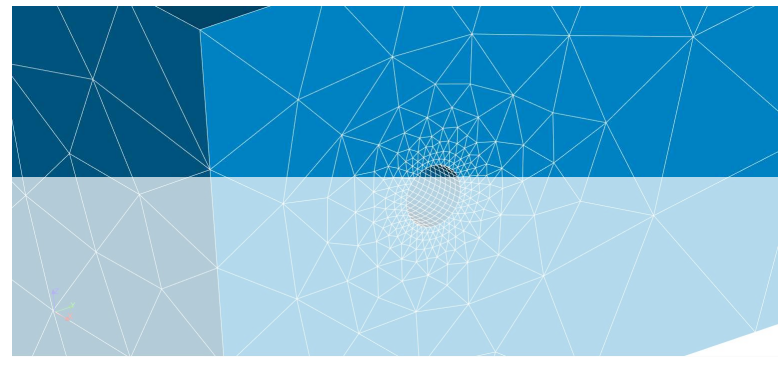

(d) Coupling to CFD solver

Figure 2: steps to perform FSI with MBDyn
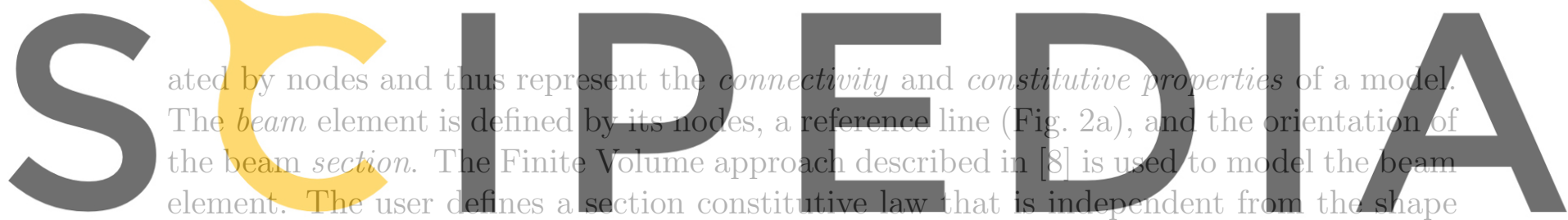

of the beam itself (Fig. 2b), thus the aerodynamic aspects and the structural aspects are

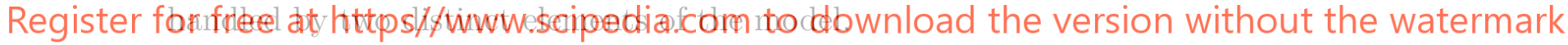

In MBDyn, it is possible to exchange kinematic and dynamic information and to steer

the multi-body simulation from an external software (left side of Fig. 1). This information can be exchanged directly on the nodes or on a cloud of external points, used for example to define an interface geometry in FSI problems (e.g. the mesh in Fig. 2c). The mapping consists in a constant matrix that computes the movement of the external points as functions of those of the nodes, based on the Moving Least Squares (MLS) technique [12]. This feature takes care of the topological inconsistency between the nodes and the interface, thus simplifying the mapping at the wet surface, as the interface mesh can be the same on both sides (Fig. 2d).

\section{2 preCICE Features}

The Open Source library preCICE aims at coupling existing solvers in a partitioned black-box manner [6]: only minimal information about each solver is required; the connection only involves the interface nodes.

In a nutshell, preCICE simply affects the input and observes the output of the solvers 
(called participants). The required data and control elements are accessed using an adapter (right side of Fig. 1).

The actions required to perform a coupled simulation involve: the set up of the communication between the participants, the computation of the mapping of data between meshes, the implementation of the coupling strategy, the verification of convergence, and the steering of the simulation.

\subsection{Adapter Configuration}

The adapter has been implemented in $\mathrm{C}++$ and is currently available from GitLab ${ }^{3}$.

The code is conceptually divided in two classes. The main class implements the functions given by the preCICE interface and has access to the other class, which takes care of all the aspects regarding MBDyn.

Some data are needed in order to perform a simulation. Such data are stored in a JSON file given as input. This information concerns:

- setup: communication with preCICE and MBDyn, mesh location for coupling and mapping
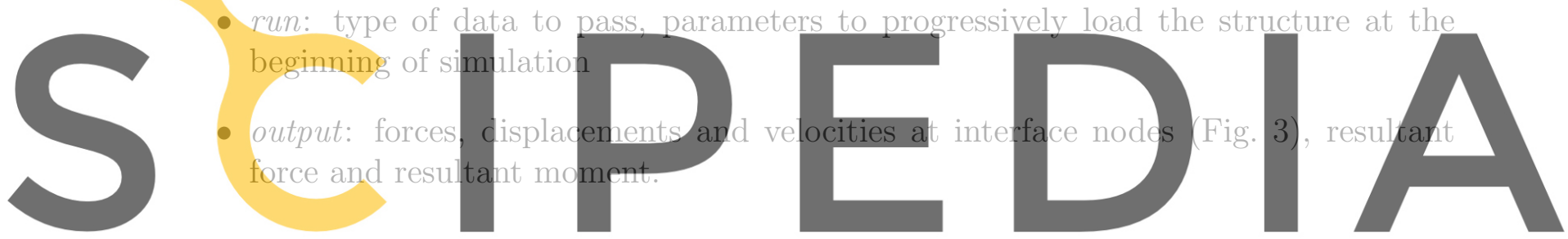

Register for free at https//www.scipedia.com to download the version without the watermark

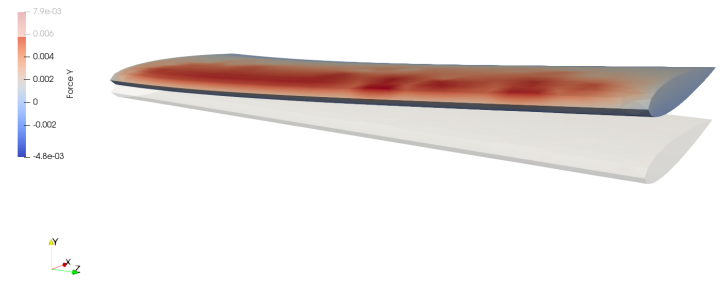

(a) Forces

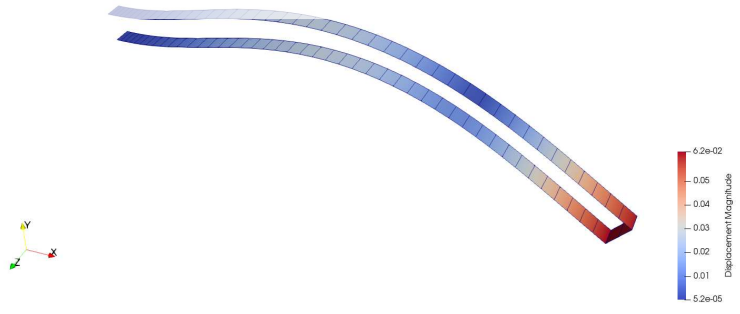

(b) Displacements

Figure 3: Simulation output

\footnotetext{
${ }^{3}$ https://public.gitlab.polimi.it/DAER/mbdyn/-/wikis/preCICE-MBDyn-adapter
} 


\section{VALIDATION OF THE COMPONENTS}

In order to validate the coupling adapter developed, we simulated some test cases to confirm the correctness of the implementation.

The three Turek-Hron FSI test cases described in [14] are a set of well-known benchmarks in FSI literature. They are characterized by the same domain (a circular cylinder with a trailing flap) and the same fluid properties. Differences are only related to fluid velocity and structural properties (in particular the density, $\rho$, and Young's modulus, $E$ ).

Besides, they are all characterized by a high mass ratio $M=\frac{\rho_{F}}{\rho_{S}}$ between the fluid and structural density, which is known to lead to algorithmic instability of the coupled system (e.g. $[7,11])$.

All the presented results are compared to the "reference" data (i.e. defined as almost grid-independent in [14]) obtained using a fully implicit monolithic ALE-FEM method with a fully coupled multigrid solver.

\subsection{Validation of the Structural Part}

The tests named CSM in [14] are used to fit the structural part. The beam is loaded only with gravitational force $\vec{g}=(0, g)=2 \mathrm{~ms}^{-2}$. Tests CSM1 and CSM2 are steady state solutions at different Young's modulus, while CSM3 is a time dependent solution

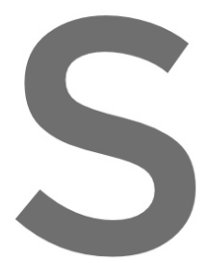
starting from the und

An MBDyn cantile been developed, as $(w \times h)$; the geometric length of the beam.
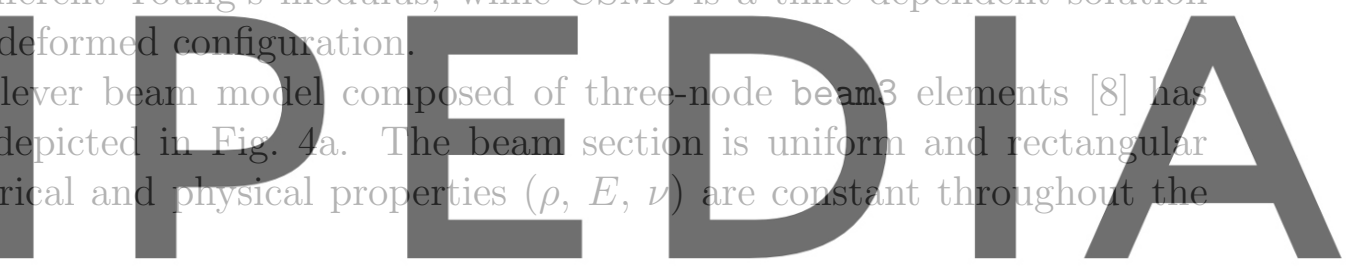

The inertia of the structure is provided by 2 rigid body elements attached to the

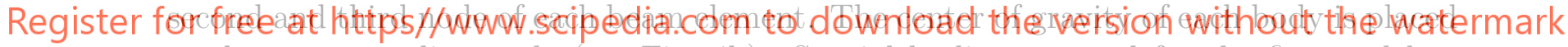
at the corresponding node (see Fig. 4b). Special bodies are used for the first and last element, with the correct mass, inertia moments, and center of mass location.

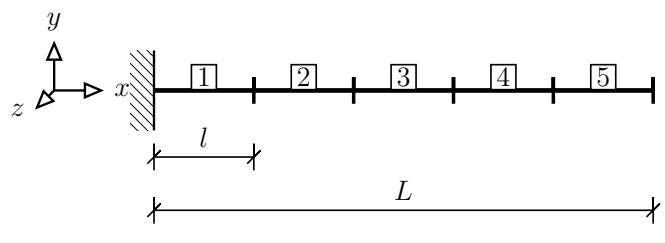

(a) Cantilever made of 5 beam elements

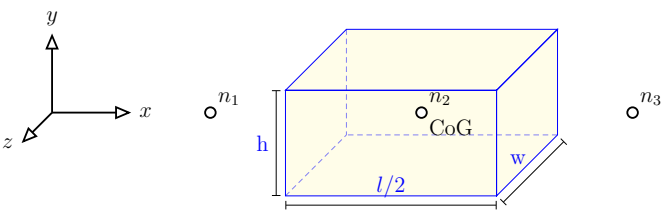

(b) Body attached to node 2 of the beam

The main parameter considered in the analysis is the number of beam elements: tests with 4 or 5 elements give results closest to the benchmark (see Table 1), while a higher number of elements makes the structure more flexible. 
Table 1: Comparison of tip displacement (in $\mathrm{mm}$ )

\begin{tabular}{c|cc|cc|ccc} 
& \multicolumn{2}{|c|}{ CSM1 } & \multicolumn{2}{c|}{ CSM2 } & \multicolumn{3}{c}{ CSM3 } \\
\hline \# el. & $u_{x}$ & $u_{y}$ & $u_{x}$ & $u_{y}$ & $u_{x}$ & $u_{y}$ & $\mathrm{f}[\mathrm{Hz}]$ \\
\hline 4 & -7.28 & -66.69 & -0.47 & -17.13 & $-14.46 \pm 14.46$ & $-65.10 \pm 65.82$ & 1.125 \\
\hline 5 & -7.73 & -68.68 & -0.51 & -17.67 & $-15.29 \pm 15.29$ & $-66.69 \pm 68.11$ & 1.104 \\
\hline 10 & -8.74 & -72.90 & -0.58 & -18.83 & $-17.57 \pm 17.57$ & $-70.82 \pm 71.20$ & 1.062 \\
\hline \hline ref. & -7.19 & -66.10 & -0.47 & -16.97 & $-14.31 \pm 14.31$ & $-63.61 \pm 65.16$ & 1.099 \\
\hline
\end{tabular}

\subsection{Validation of the Fluid Part}

The fluid part has been modeled with OpenFOAM, using a hexahedral mesh with different levels of refinement (Fig. 5).
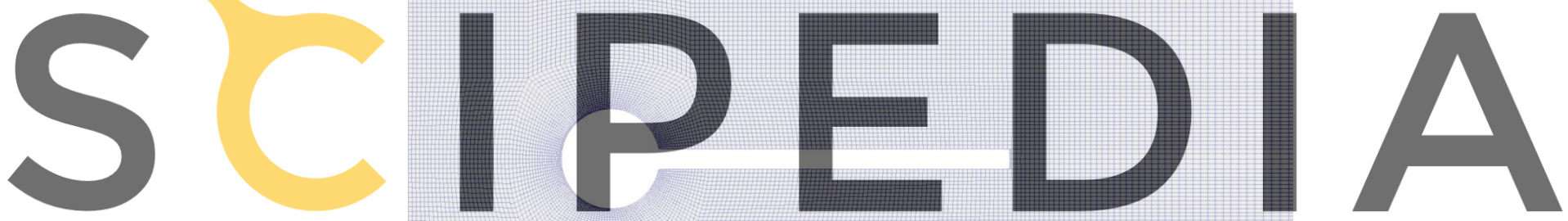

Register for free at https//www.scipedia.com to download the version without the watermark

Figure 5: Fluid mesh used for CFD and FSI simulations

The tests named CFD in [14] have been used to understand the fitness of the fluid part. All 3 cases share the same fluid properties, a parabolic inlet profile and a rigid domain. They differ only in average fluid velocity, with values of $0.2,1$ and $2 \mathrm{~m} \mathrm{~s}^{-1}$. The results are shown in Table 2: at low fluid speed the impact of the fineness of the mesh is low or negligible, whereas it becomes relevant for CFD3, characterized by alternating vortices developing downstream of the structure. 
Table 2: Comparison of lift and drag (in $\mathrm{N} \mathrm{m}^{-1}$ )

\begin{tabular}{c|cc|cc|ccc} 
& \multicolumn{2}{|c|}{ CFD1 } & \multicolumn{2}{c|}{ CFD2 } & \multicolumn{3}{c}{ CFD3 } \\
\hline size & drag & lift & drag & lift & drag & lift & $\mathrm{f}[\mathrm{Hz}]$ \\
\hline $25 \mathrm{k}$ & 14.28 & 1.11 & 139.49 & 10.77 & $442.53 \pm 4.75$ & $-17.39 \pm 384.22$ & 4.38 \\
\hline $49 \mathrm{k}$ & 14.28 & 1.11 & 137.34 & 10.46 & $441.45 \pm 5.17$ & $-15.51 \pm 412.03$ & 4.41 \\
\hline \hline ref. & 14.29 & 1.12 & 136.7 & 10.53 & $439.45 \pm 5.62$ & $-11.89 \pm 437.81$ & 4.3956 \\
\hline
\end{tabular}

\section{TEST CASES}

Once the solid and the fluid models have been assessed, we considered the FSI test cases described in [14].

\subsection{General Set-Up}

For each simulation we used the staggered implicit coupling algorithm implemented in preCICE, with the structural component as the first participant and the fluid component as the second one. We considered the IQN-ILS algorithm $([2,5])$ and a filter in order to

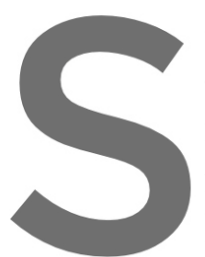
drop nearly dependent error of $10^{-4}$ for both much faster than the forces, which thus be has yet to be assessed.
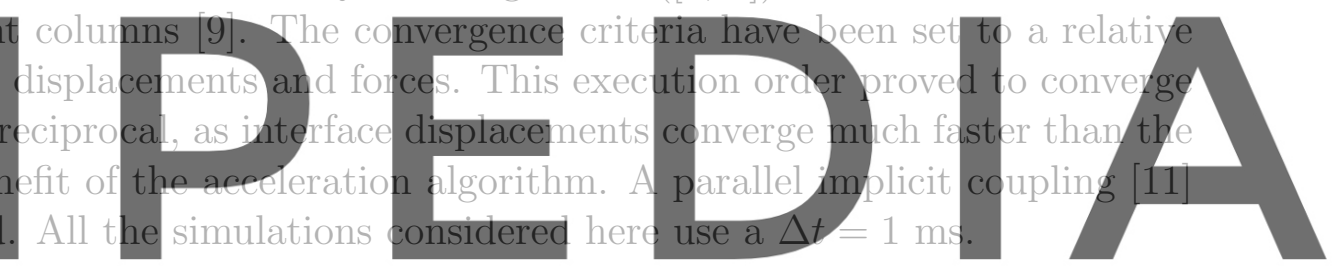

Register for free ${ }^{\text {FSI } 1}$

The first test case is characterized by a mass ratio $M=1$ and an average Reynolds number $R e=20$. The position of the beam is not symmetric such that the lift is not zero [17]. The final solution is steady (see Figure 6). It is nevertheless interesting because its high mass ratio and low fluid velocity make the interaction stronger and the convergence sometimes more difficult in partitioned algorithms. The results, in terms of tip displacement and forces on the structure, are shown in Table 3 and compared with the reference in $[14]$.

The coupled simulation converged with an average number of 2.8 iterations. There is no significant difference with respect to the mesh size, and the results are close to the reference.

\section{$4.3 \quad$ FSI2}

The second test case is characterized by a mass ratio $M=0.1$ and an average Reynolds number $R e=100$. This test case is fully oscillating while the same problem, considering the structure rigid (CFD2 in [14]) is steady: for this reason, it is considered an excellent 


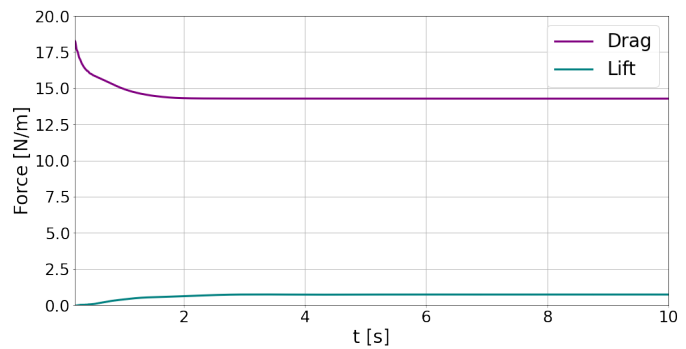

(a) Forces

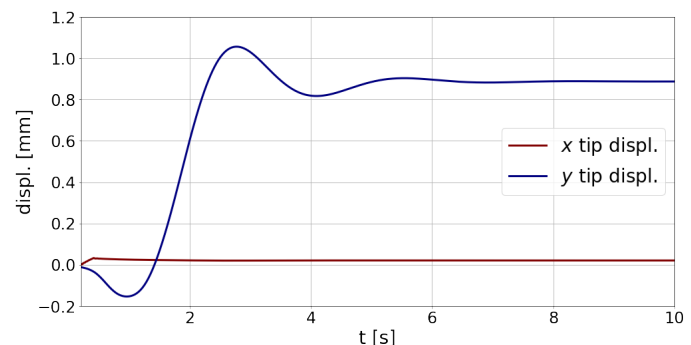

(b) Tip displacements

Figure 6: FSI1 Simulation: 5 beams - 49k cells

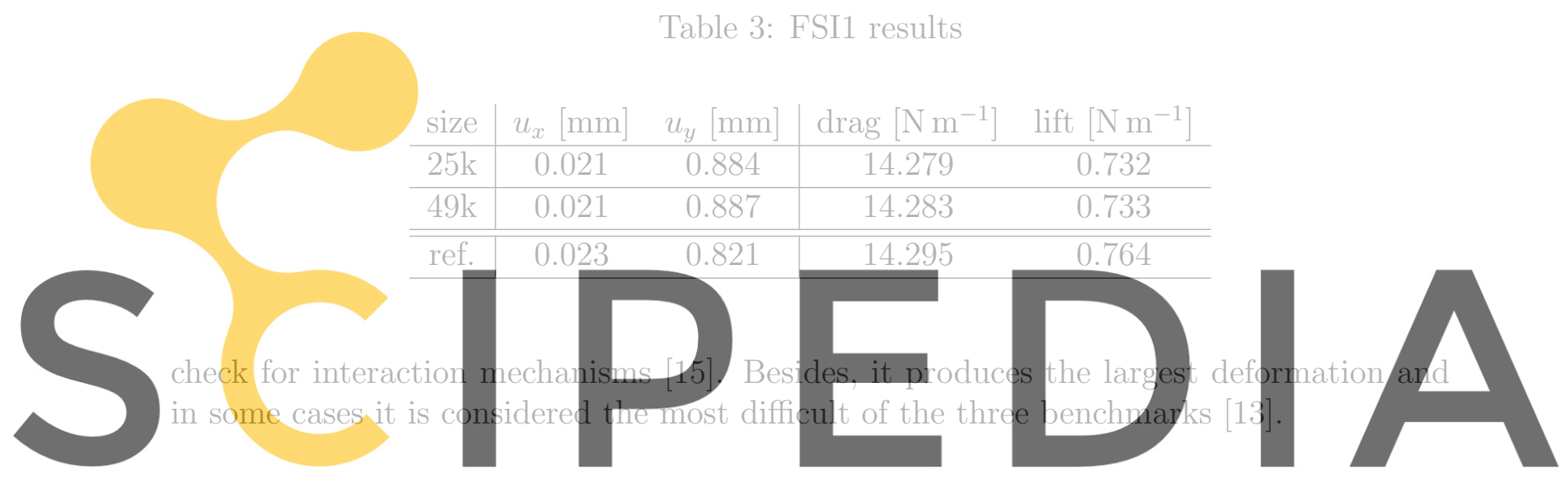

Register for free at https//www.scipedia.com to down'load the version without the watermark
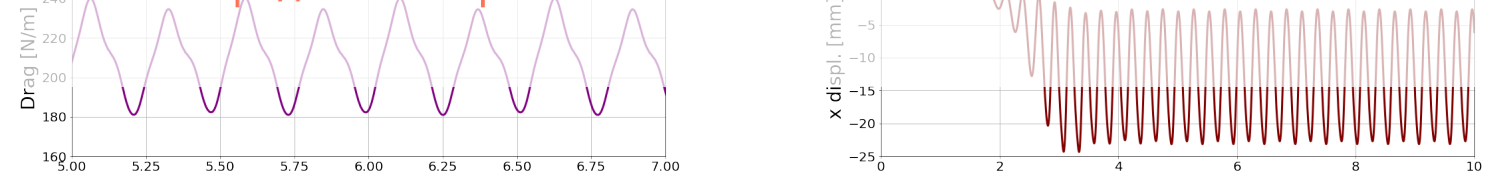

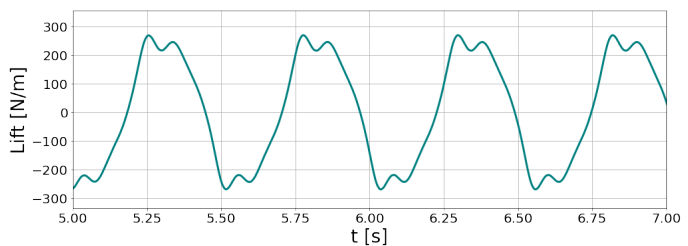

(a) Forces (detail)

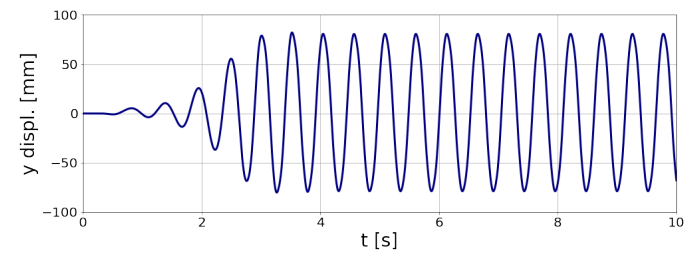

(b) Tip displacements

Figure 7: FSI2 Simulation: 5 beams - 49k cells

The behavior of the structure in terms of tip displacement (mean value, amplitude and frequency) is shown in Table 4, while drag and lift on the whole structure are shown in 
Table 5 .

Table 4: FSI2 results (displacements)

\begin{tabular}{c|cc|cc} 
size & $u_{x}[\mathrm{~mm}]$ & $\mathrm{f}[\mathrm{Hz}]$ & $u_{y}[\mathrm{~mm}]$ & $\mathrm{f}[\mathrm{Hz}]$ \\
\hline $25 \mathrm{k}$ & $-12.86 \pm 10.05$ & 3.84 & $0.91 \pm 79.72$ & 1.92 \\
\hline $49 \mathrm{k}$ & $-10.66 \pm 7.72$ & 3.79 & $1.67 \pm 74.19$ & 1.90 \\
\hline \hline ref. & $-14.58 \pm 12.44$ & 3.86 & $1.23 \pm 80.6$ & 1.93 \\
\hline
\end{tabular}

\section{Table 5: FSI2 results (forces)}

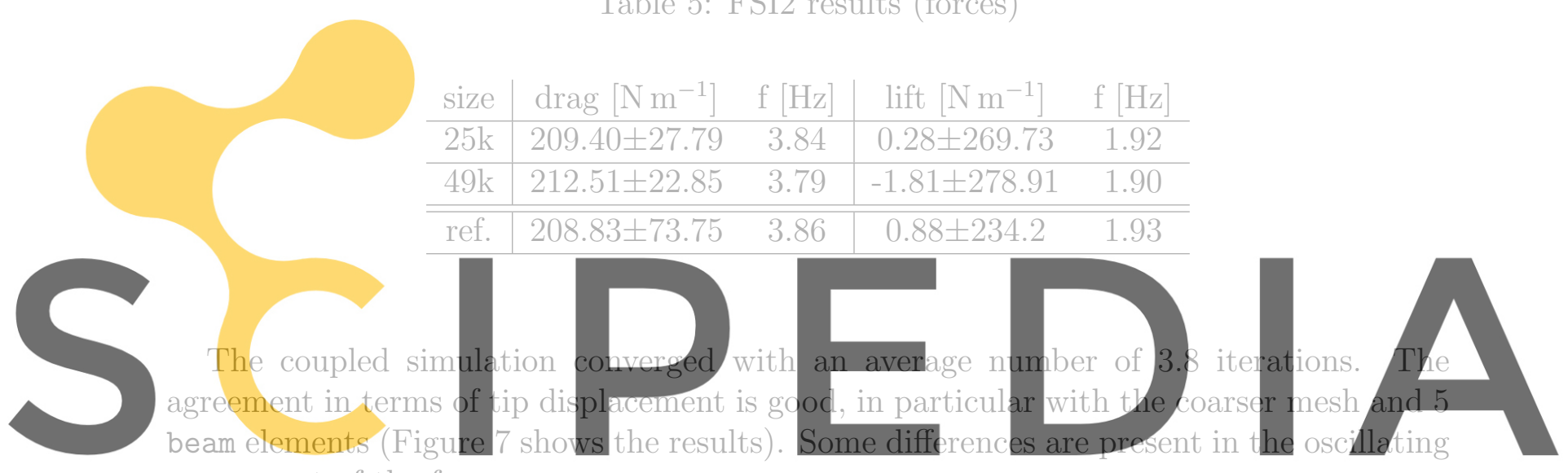

component of the forces.

Register for free at https//www.scipedia.com to download the version without the watermark

\subsection{FSI3}

The last test case is characterized by a mass ratio $M=1$ and an average Reynolds number $R e=200$. This case has been widely studied and a review of results obtained with different approaches (monolithic or partitioned, with different solution strategies) can be found in [16].

The behavior of the structure in terms of tip displacement (mean value, amplitude and frequency) is shown in Table 6 , while drag and lift on the whole structure are shown in Table 7.

The coupled simulation converged with an average number of 3.5 iterations (Figure 8 shows the results). As pointed out in [16], different solution approaches lead to differences of up to $50 \%$ for the drag and lift values, and up to $10 \%$ for the displacement values.

In our simulations, different discretizations lead to quite different solutions and the trade-off between the efficiency of a coarser mesh and the accuracy of a finer one is apparent. Besides, other simulation parameters have an impact on the results: e.g. the structural damping of the elastic beam changes the amplitude of the $y$ component of the 

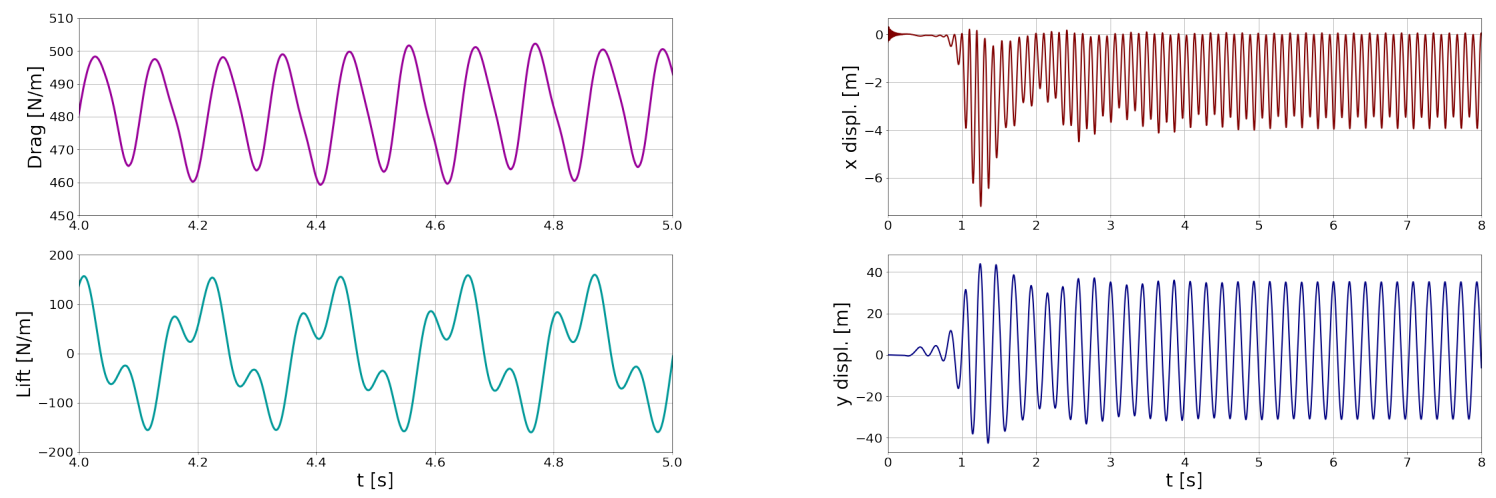

(a) Forces (detail)

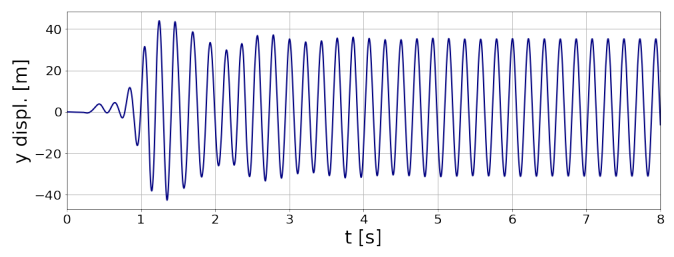

(b) Tip displacements

Figure 8: FSI3 Simulation: 4 beams - 49k cells

Table 6: FSI3 results (displacements)
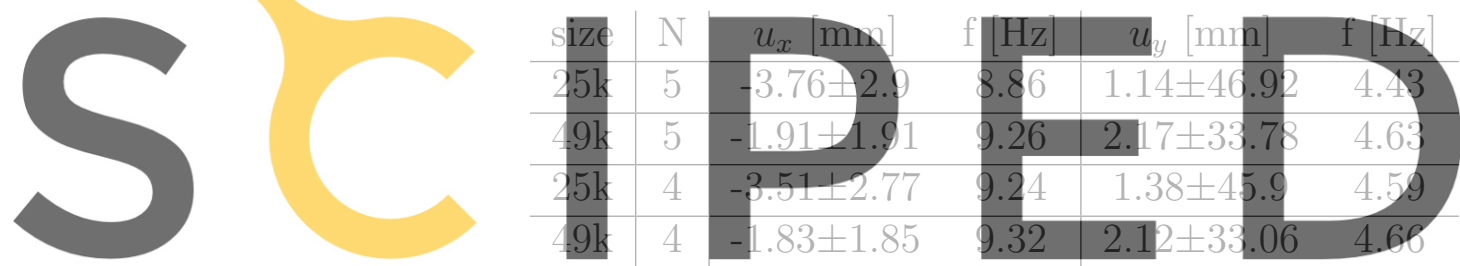

\begin{tabular}{l|ll|ll} 
ref. & $-2.69 \pm 2.53$ & 10.9 & $1.48 \pm 34.38$ & 5.3
\end{tabular}

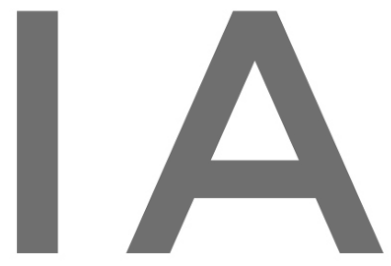

Register for free at https//www.scipedia.com to download the version without the watermark

Table 7: FSI3 results (forces)

\begin{tabular}{c|c|cc|cc} 
size & $\mathrm{N}$ & $\operatorname{drag}\left[\mathrm{N} \mathrm{m}^{-1}\right]$ & $\mathrm{f}[\mathrm{Hz}]$ & lift $\left[\mathrm{N} \mathrm{m}^{-1}\right]$ & $\mathrm{f}[\mathrm{Hz}]$ \\
\hline $25 \mathrm{k}$ & 5 & $515.54 \pm 35.07$ & 8.87 & $-1.28 \pm 78.65$ & 4.43 \\
\hline $49 \mathrm{k}$ & 5 & $481.24 \pm 17.64$ & 9.26 & $-0.48 \pm 164.52$ & 4.63 \\
\hline $25 \mathrm{k}$ & 4 & $502.62 \pm 30.03$ & 9.24 & $1.51 \pm 97.31$ & 4.59 \\
\hline $49 \mathrm{k}$ & 4 & $481.12 \pm 19.07$ & 9.32 & $-0.20 \pm 157.64$ & 4.66 \\
\hline \hline ref. & & $457.3 \pm 22.66$ & 10.9 & $2.22 \pm 149.78$ & 5.3 \\
\hline
\end{tabular}

tip displacement and the oscillation frequency. This last result appears to be lower than most of other studies in literature and might require a better tuning of the simulation of parameters. 


\section{CONCLUSIONS}

The proposed adapter shows a good agreement with the considered benchmarks. Despite considering quite simple meshes, most results reported here look close to the reference values, showing that the current implementation proved to be robust with respect to strong interaction (high mass ratio). Thorough assessment in compressible regime (e.g. aeroelasticity) is underway.

Even though it must be thoroughly tested in realistic scenarios, the overall set-up proved to be flexible, widely configurable and tunable. In particular the ability to progressively load the structure at the beginning of the simulation turned out to be a very useful feature.

The adapter exploits the features provided by MBDyn for partitioned cosimulations and, via the common interfacer preCICE, it can connect MBDyn to virtually any solver, to perform FSI (fluid-solid interaction) and possibly hybrid multi-body full finite element simulations. It still requires some minor improvements, e.g. to use nearest-projection mappings in preCICE.

The code is independent of the MBDyn source code, which makes it easily extensible and maintainable.

\section{References}

[1] Olivier A Bauchau et al. "Validation of flexible multibody dynamics beam formulations using benchmark problems". In: Multibody system dynamics 37.1 (2016), pp. 29-48.

[2] David Blom et al. "A Review on Fast Quasi-Newton and Accelerated Fixed-Point Iterations for Partitioned Fluid-Structure Interaction Simulation". In: Advances in Computational Fluid-Structure Interaction and Flow Simulation. Springer, 2016, pp. 257-269.

[3] Hans-Joachim Bungartz et al. "preCICE-a fully parallel library for multi-physics surface coupling". In: Computers $\&$ Fluids 141 (2016), pp. 250-258.

[4] Alessandro Cocco et al. "Simulation of Tiltrotor Maneuvers by a Coupled MultibodyMid Fidelity Aerodynamic Solver". In: 46th European Rotorcraft Forum (ERF 2020). 2020, pp. 1-8.

[5] J Degroote et al. "An interface quasi-Newton algorithm for partitioned simulation of fluid-structure interaction". In: International Workshop on Fluid-Structure Interaction. Theory, Numerics and Applications. kassel university press GmbH. 2009, p. 55 .

[6] Joris Degroote, Klaus-Jürgen Bathe, and Jan Vierendeels. "Performance of a new partitioned procedure versus a monolithic procedure in fluid-structure interaction". In: Computers \& Structures 87.11-12 (2009), pp. 793-801.

[7] Joris Degroote et al. "Stability of a coupling technique for partitioned solvers in FSI applications". In: Computers \&5 Structures 86.23-24 (2008), pp. 2224-2234. 
[8] Gian Luca Ghiringhelli, Pierangelo Masarati, and Paolo Mantegazza. "Multibody implementation of finite volume $\mathrm{C}^{0}$ beams". In: AIAA journal 38.1 (2000), pp. 131138.

[9] Rob Haelterman et al. "Improving the performance of the partitioned QN-ILS procedure for fluid-structure interaction problems: Filtering". In: Computers $\& 5$ Structures 171 (2016), pp. 9-17.

[10] Pierangelo Masarati, Marco Morandini, and Paolo Mantegazza. "An efficient formulation for general-purpose multibody/multiphysics analysis". In: Journal of Computational and Nonlinear Dynamics 9.4 (2014).

[11] Miriam Mehl et al. "Parallel coupling numerics for partitioned fluid-structure interaction simulations". In: Computers \& Mathematics with Applications 71.4 (2016), pp. 869-891.

[12] Giuseppe Quaranta, Pierangelo Masarati, and Paolo Mantegazza. "A conservative mesh-free approach for fluid-structure interface problems". In: International Conference for Coupled Problems in Science and Engineering, Greece. 2005.

[13] Thomas Richter and Thomas Wick. "On time discretizations of fluid-structure interactions". In: Multiple Shooting and Time Domain Decomposition Methods. Springer, 2015, pp. 377-400.

[14] Stefan Turek and Jaroslav Hron. "Proposal for numerical benchmarking of fluidstructure interaction between an elastic object and laminar incompressible flow". In: Fluid-structure interaction. Springer, 2006, pp. 371-385.

[15] Stefan Turek, Jaroslav Hron, and Mudassar Razzaq. "Numerical benchmarking of fluid-structure interaction between elastic object and laminar incompressible flow". In: Universitätsbibliothek Dortmund (2010).

[16] Stefan Turek et al. "Numerical Benchmarking of Fluid-Structure Interaction: A Comparison of Different Discretization and Solution Approaches". In: vol. 73. Sept. 2010, pp. 413-424. ISBN: 978-3-642-14205-5. DOI: 10 . 1007 /978-3-642-14206$2 \_15$.

[17] Stefan Turek et al. "Numerical Simulation and Benchmarking of a Monolithic Multigrid Solver for Fluid-Structure Interaction Problems with Application to Hemodynamics". In: vol. 73. Sept. 2010, pp. 193-220. ISBN: 978-3-642-14205-5. DOI: 10. 1007/978-3-642-14206-2_8.

[18] Benjamin Uekermann et al. "Official preCICE adapters for standard open-source solvers". In: Proceedings of the 7th GACM Colloquium on Computational Mechanics for Young Scientists from Academia. 2017. 